\title{
AN INTERPOLATION THEOREM AND A SHARP FORM OF A MULTILINEAR FRACTIONAL INTEGRATION THEOREM
}

\author{
JONG-GUK BAK
}

(Communicated by Andrew M. Bruckner)

\begin{abstract}
We prove a sharp interpolation theorem for Orlicz spaces with the Luxemburg norm. As a corollary we obtain a sharp form of an exponential integrability theorem, due to Grafakos, for the multilinear fractional integration operator. This generalizes a theorem of Adams.
\end{abstract}

\section{INTRODUCTION}

In his 1988 paper [A] Adams proved a sharp form of certain limiting cases of the Sobolev embedding theorem by first establishing the following exponential integrability theorem for the fractional integration operator $I_{\alpha}$ (Riesz potential of order $\alpha)$ defined by $I_{\alpha}(f)(x)=\int_{\mathbf{R}^{n}} f(x-y)|y|^{\alpha-n} d y \quad(0<\alpha<n)$. The symbol $p^{\prime}=\frac{p}{p-1}$ denotes the conjugate exponent to $p, w_{n-1}$ the surface area of the unit sphere in $\mathbf{R}^{n}$, and $|\Omega|$ the Lebesgue measure of the set $\Omega \subset \mathbf{R}^{n}$.

Theorem A (Adams [A]). For $p \in(1, \infty)$ and $\alpha=\frac{n}{p}$, there is a constant $c_{0}=c_{0}(p)$ depending only on $p$ such that for all $f \in L^{p}\left(\mathbf{R}^{n}\right)$ with support contained in a domain $\Omega$ in $\mathbf{R}^{n},|\Omega|<\infty$,

$$
\frac{1}{|\Omega|} \int_{\Omega} \exp \left(\frac{n}{w_{n-1}}\left|\frac{I_{\alpha}(f)(x)}{\|f\|_{p}}\right|^{p^{\prime}}\right) d x \leq c_{0} .
$$

Furthermore, (1) fails if $\frac{n}{w_{n-1}}$ is replaced by a larger constant.

Grafakos [G] extended this result to cover a multilinear analog of $I_{\alpha}$, namely, the operator $I_{\alpha}\left(f_{1}, \ldots, f_{K}\right)$ defined by

$$
I_{\alpha}\left(f_{1}, \ldots, f_{K}\right)(x)=\int_{\mathbf{R}^{n}} f_{1}\left(x-\theta_{1} y\right) \cdots f_{K}\left(x-\theta_{K} y\right)|y|^{\alpha-n} d y,
$$

where $\theta_{j} \in \mathbf{R} \backslash 0,1 \leq j \leq K$. He proved the following theorem, which may be called a multilinear fractional integration theorem.

Theorem B (Grafakos [G]). Let $p \in(1, \infty), \frac{1}{p}=\sum_{j=1}^{K} \frac{1}{p_{j}}, p_{j} \in(1, \infty]$, and $\alpha=\frac{n}{p}$. Assume the real numbers $\theta_{j} \neq 0$ are distinct. Let $B$ be a ball in $\mathbf{R}^{n}$,

Received by the editors May 6, 1992.

1991 Mathematics Subject Classification. Primary 42B99, 26A33, 46B70. 
and let $f_{j} \in L^{p_{j}}$ be supported in $B$. Then for any $\gamma<1$, there exists a constant $C_{0}(\gamma)$ depending only on $n, \alpha, \theta_{j}$ 's, and $\gamma$ such that

$$
\frac{1}{|B|} \int_{B} \exp \left(\frac{n}{w_{n-1}} \gamma\left|\frac{L I_{\alpha}\left(f_{1}, \ldots, f_{K}\right)(x)}{\left\|f_{1}\right\|_{p_{1}} \cdots\left\|f_{K}\right\|_{p_{K}}}\right|^{p^{\prime}}\right) d x \leq C_{0}(\gamma)
$$

where $L=\prod_{j=1}^{K}\left|\theta_{j}\right|^{n / p_{j}}$. Furthermore, (2) fails if $\gamma>1$.

Note that Theorem B leaves the case $\gamma=1$ open. The proof of (2) was based on complicated estimates involving properties of the multisublinear maximal function defined by

$$
M\left(f_{1}, \ldots, f_{K}\right)(x)=\sup _{r>0} \frac{1}{|B(0, r)|} \int_{B(0, r)}\left|f_{1}\left(x-\theta_{1} y\right) \cdots f_{K}\left(x-\theta_{K} y\right)\right| d y,
$$

where $B(0, r)$ is the Euclidean ball of radius $r$ centered at the origin in $\mathbf{R}^{n}$. (Here $\theta_{j} \in \mathbf{R}^{n} \backslash 0$ are assumed to be distinct.) The purpose of this note is to show that the end point case $\gamma=1$ of (2) is actually an easy consequence of Theorem A, once we establish a sharp form of a multilinear interpolation theorem for Orlicz spaces (with the Luxemburg norm). We will state and prove this interpolation result in $\S 2$. Our observations may be combined with the known results and stated as follows:

Theorem 1. Let $K \geq 1, p \in(1, \infty), \alpha=\frac{n}{p}, \frac{1}{p}=\sum_{j=1}^{K} \frac{1}{p_{j}}$, and $p_{j} \in(1, \infty]$. Then there exists a constant $c_{0}=c_{0}(p)$ depending only on $p$ such that for all $f_{j} \in L^{p_{j}}\left(\mathbf{R}^{n}\right)$ with support contained in a domain $\Omega$ in $\mathbf{R}^{n},|\Omega|<\infty$,

$$
\frac{1}{|\Omega|} \int_{\Omega} \exp \left(\frac{n}{w_{n-1}}\left|\frac{L I_{\alpha}\left(f_{1}, \ldots, f_{K}\right)(x)}{\left\|f_{1}\right\|_{p_{1}} \cdots\left\|f_{K}\right\|_{p_{K}}}\right|^{p^{\prime}}\right) d x \leq c_{0},
$$

where $L=\prod_{j=1}^{K}\left|\theta_{j}\right|^{n / p_{j}}$. Furthermore, (3) fails if $\frac{n}{w_{n-1}}$ is replaced by a larger constant.

Remark. The constant $c_{0}$ in (3) is in fact identical to the one appearing in (1). So it depends only on the ratio $n / \alpha=p$. On the other hand, note that the constant $C_{0}(\gamma)$ in (2) depends on the other parameters also. In particular, $C_{0}(\gamma) \rightarrow \infty$, as $\gamma \rightarrow 1^{-}$.

Theorem 1 will be proved in $\S 3$.

\section{A MULTILINEAR INTERPOLATION THEOREM FOR ORLICZ SPACES}

Let $(X, \mathscr{M}, \mu)$ be a $\sigma$-finite measure space. A convex function $Q:[0, \infty) \rightarrow$ $[0, \infty]$ is called a Young's function if $Q(0)=0$ and $Q$ is not identically 0 or $\infty$ on $(0, \infty)$. Given a measurable function $f$ on $X$ we let

$$
\begin{array}{ll}
\|f\|_{Q}=\inf \left\{l>0: \int Q\left(\frac{|f|}{l}\right) d \mu \leq 1\right\} & \text { (Luxemburg norm), } \\
\|f\|_{Q}^{*}=\sup _{\|g\|_{Q^{*} \leq 1}\left|\int f g d \mu\right|} \mid \text { (Orlicz norm), }
\end{array}
$$

where $Q^{*}$ denotes Young's complementary function of $Q$ (defined by $Q^{*}(t)=$ $\sup _{s \geq 0}[s t-Q(s)]$ for $\left.t \geq 0\right)$. $\|f\|_{Q}$ and $\|f\|_{Q}^{*}$ are equivalent norms on the 
Orlicz space $L^{Q}(X)=\left\{f:\|f\|_{Q}<\infty\right\}$. The letters $\Phi, Q$, and $R$ (with subscripts) will stand for Young's functions. Given a Young's function $Q$ its (generalized) inverse is defined by $Q^{-1}(t)=\sup \{l \geq 0: Q(l)<t\}$ (for $t>0$ ). We will write $R_{\ell}$ for Young's complementary function of $Q_{\ell}, \ell=0,1 . R_{s}$ will denote the intermediate function defined to be the inverse of the function $R_{s}^{-1}(t)=\left(R_{0}^{-1}(t)\right)^{1-s}\left(R_{1}^{-1}(t)\right)^{s}$ for $0 \leq s \leq 1$. For more details on Orlicz spaces see $[\mathrm{KR}, \mathrm{R}]$.

We now state our interpolation result. Note that all the norms in the statement of Theorem 2 are the Luxemburg norms (see the remark after the proof of the theorem). Let $\left(X_{j}, \mathscr{M}_{j}, \mu_{j}\right)$ be $\sigma$-finite measure spaces and

$$
T: L^{\Phi_{1, \ell}}\left(X_{1}\right) \times \cdots \times L^{\Phi_{K, \ell}}\left(X_{K}\right) \rightarrow L^{Q_{\ell}}(X)
$$

denote a multilinear operator. $M_{\ell}$ will denote constants independent of the functions $f_{j}$.

Theorem 2. Let $T$ be a multilinear operator such that

$$
\left\|T\left(f_{1}, \ldots, f_{K}\right)\right\|_{Q_{\ell}} \leq M_{\ell} \prod_{j=1}^{K}\left\|f_{j}\right\|_{\Phi_{j, \ell}}, \quad \ell=0,1
$$

If $\Phi_{j, s}^{-1}(t)=\left(\Phi_{j, 0}^{-1}(t)\right)^{1-s} \cdot\left(\Phi_{j, 1}^{-1}(t)\right)^{s}, 1 \leq j \leq K, 0 \leq s \leq 1$, and $Q_{s}^{+}=\left(R_{s}\right)^{*}$, then

$$
\left\|T\left(f_{1}, \ldots, f_{K}\right)\right\|_{Q_{s}^{+}} \leq M_{0}^{1-s} M_{1}^{s} \prod_{j=1}^{K}\left\|f_{j}\right\|_{\Phi_{j, s}},
$$

where $f_{j}$ are (integrable) simple functions.

The following lemma is stated on p. 135 of $[\mathrm{KR}]$ in the case when $\mu$ is finite. Essentially the same proof can be used to show that it is valid when $\mu$ is $\sigma$-finite and $f \in L^{1} \cap L^{Q}(\mu)$. We wish to thank Steve Bellenot for helpful conversations about the lemma.

Lemma 3. If $Q$ and $Q^{*}$ are continuous and $f \in L^{1} \cap L^{Q}(\mu)$ then

$$
\|f\|_{Q}=\sup \left\{\left|\int f g d \mu\right|:\|g\|_{Q^{*}}^{*} \leq 1\right\} .
$$

Proof of Theorem 2. As was observed in [R] we may assume all of our Young's functions are continuous and strictly increasing. By Lemma 3 we have

$$
\left\|T\left(f_{1}, \ldots, f_{K}\right)\right\|_{Q_{s}^{+}}=\sup \left\{\left|\int T\left(f_{1}, \ldots, f_{K}\right) g d \mu\right|:\|g\|_{R_{s}}^{*} \leq 1\right\},
$$

if the $f_{j}$ 's are integrable simple functions. Fix a number $s \in(0,1)$. For $1 \leq j \leq K, \ell=0,1$, and $z \in \mathbf{C}$, let $f_{j}$ and $g$ be (integrable) simple functions with $\left\|f_{j}\right\|_{\Phi_{j, s}}=1$ and $\|g\|_{R_{s}}^{*}=1$. Then it suffices to show that $|I|=$ $\left|\int T\left(f_{1}, \ldots, f_{K}\right) g d \mu\right| \leq M_{0}^{1-s} M_{1}^{s}$. Let $\alpha_{j, \ell}=\Phi_{j, \ell}^{-1}, \alpha_{j, z}(t)=\left(\alpha_{j, 0}(t)\right)^{1-z}$. $\left(\alpha_{j, 1}(t)\right)^{z}, \beta_{\ell}=R_{\ell}^{-1}$, and $\beta_{z}(t)=\left(\beta_{0}(t)\right)^{1-z} \cdot\left(\beta_{1}(t)\right)^{z}$. Define

$$
f_{j, z}=\alpha_{j, z}\left(\Phi_{j, s}\left(\left|f_{j}\right|\right)\right) \cdot e^{i \arg \left(f_{j}\right)} \text {. }
$$


Let $\varepsilon>0$. Since $R_{s}$ is continuous by assumption, we have

$$
1=\|g\|_{R_{s}}^{*}=\inf _{k>0} \frac{1}{k}\left(1+\int R_{s}(k|g|) d \mu\right)
$$

(see [KR, p. 92]). Hence there exists a number $k_{s, \varepsilon} \in(0, \infty)$ such that

$$
\frac{1}{k_{s, \varepsilon}}\left(1+\int R_{s}\left(k_{s, \varepsilon}|g|\right)\right) \leq 1+\varepsilon .
$$

Now define

$$
g_{z}=\frac{1}{k_{s, \varepsilon}} \beta_{z}\left(R_{s}\left(k_{s, \varepsilon}|g|\right)\right) \cdot e^{i \arg (g)} .
$$

Then $I(z)=\int T\left(f_{1, z}, \ldots, f_{K, z}\right) g_{z} d \mu$ can be shown to be continuous in the strip $\{z \in \mathbf{C}: 0 \leq \operatorname{Re} z \leq 1\}$ and analytic in $\{z \in \mathbf{C}: 0<\operatorname{Re} z<1\}$ (see $[R]$ ). By Hölder's inequality (6),

$$
\begin{aligned}
|I(i y)| & \leq\left\|T\left(f_{1, i y}, \ldots, f_{K, i y}\right)\right\|_{Q_{0}} \cdot\left\|g_{i y}\right\|_{R_{0}}^{*} \\
& \leq M_{0}\left\|g_{i y}\right\|_{R_{0}}^{*} \prod_{j=1}^{K}\left\|f_{j, i y}\right\|_{\Phi_{j, 0}} \quad(y \in \mathbf{R}) .
\end{aligned}
$$

Now $\int \Phi_{j, 0}\left(\left|f_{j, i y}\right|\right) d \mu_{j}=\int \Phi_{j, 0}\left(\alpha_{j, 0}\left(\Phi_{j, s}\left(\left|f_{j}\right|\right)\right)\right)=\int \Phi_{j, s}\left(\left|f_{j}\right|\right) \leq 1$, since $\left\|f_{j}\right\|_{\boldsymbol{\Phi}_{j, s}}=1$. So $\left\|f_{j, i y}\right\|_{\boldsymbol{\Phi}_{j, 0}} \leq 1$. Also,

$$
\begin{aligned}
\left\|g_{i y}\right\|_{R_{0}}^{*} & =\inf _{k>0} \frac{1}{k}\left(1+\int R_{0}\left(k\left|g_{i y}\right|\right)\right) \leq \frac{1}{k_{s, \varepsilon}}\left(1+\int R_{0}\left(k_{s, \varepsilon}\left|g_{i y}\right|\right)\right) \\
& =\frac{1}{k_{s, \varepsilon}}\left(1+\int R_{0}\left(\beta_{0}\left(R_{s}\left(k_{s, \varepsilon}|g|\right)\right)\right)\right) \\
& =\frac{1}{k_{s, \varepsilon}}\left(1+\int R_{s}\left(k_{s, \varepsilon}|g|\right)\right) \leq 1+\varepsilon .
\end{aligned}
$$

Therefore, $|I(i y)| \leq(1+\varepsilon) M_{0} \quad \forall y \in \mathbf{R}$. Similarly, $|I(1+i y)| \leq(1+\varepsilon) M_{1}$. Hence by the three lines theorem, it follows that

$$
|I|=|I(s)| \leq(1+\varepsilon) M_{0}^{1-s} M_{1}^{s}, \quad 0 \leq s \leq 1 .
$$

Since $\varepsilon>0$ was arbitrary, we conclude that $|I| \leq M_{0}^{1-s} M_{1}^{s}$.

Remark. Rao (see [R, Theorems 1, 2, and 4]) originally proved an analog of Theorem 2 with the sharp constant $M_{0}^{1-s} M_{1}^{s}$ using the Orlicz norm on both sides in place of the Luxemburg norm. Since the two norms are equivalent $\left(\|f\|_{Q} \leq\|f\|_{Q}^{*} \leq 2\|f\|_{Q}\right)$, it is easy to see that Rao's result implies (4) with the constant $M_{0}^{1-s} M_{1}^{s}$ replaced by $A M_{0}^{1-s} M_{1}^{s}$ with some constant $A>1$ (see the remark following Theorem 2 in [R]; also see [M, p. 102] in this connection).

Corollary 4. Let $T$ be a multilinear operator such that for $p_{j, \ell} \in[1, \infty]$

$$
\left\|T\left(f_{1}, \ldots, f_{K}\right)\right\|_{Q} \leq M_{\ell} \prod_{j=1}^{K}\left\|f_{j}\right\|_{p_{j, \ell}} \quad(\ell=0,1) .
$$


If $\frac{1}{p_{j}}=\frac{1}{p_{j, s}}=\frac{1-s}{p_{j, 0}}+\frac{s}{p_{j, 1}}, 1 \leq j \leq K, 0 \leq s \leq 1$, then

$$
\left\|T\left(f_{1}, \ldots, f_{K}\right)\right\|_{Q} \leq M_{0}^{1-s} M_{1}^{s} \prod_{j=1}^{K}\left\|f_{j}\right\|_{p_{j}} .
$$

Proof. Just take $Q_{\ell}=Q_{s}^{+}=Q$, and $\Phi_{j, s}(t)=t^{p_{j, s}}$ (when $p_{j, s}=\infty$, take $\Phi_{j, s}(t)=0$ for $t \leq 1$, and $=\infty$ for $t>1$ ) in Theorem 2. (In fact, in this case the arguments simplify considerably. So we may give a short direct proof with $g_{z}=g$ and

$$
f_{j, z}=\left|f_{j}\right|^{p_{j} / p_{j}(z)} \cdot e^{i \arg \left(f_{j}\right)} \quad\left(\text { if } p_{j}<\infty\right),
$$

where $\frac{1}{p_{j}(z)}=\frac{1-z}{p_{j, 0}}+\frac{z}{p_{j, 1}}$. (Let $f_{j, z}=f_{j}$, if $p_{j}=\infty$.) The rest proceeds exactly as in the proof of the Riesz-Thorin theorem (see [SW]) once we use Lemma 3 and Hölder's inequality (6).)

Corollary 5. Let $K \geq 2, p \in[1, \infty)$, where $\frac{1}{p}=\sum_{j=1}^{K} \frac{1}{p_{j}}$, and $p_{j} \in(1, \infty]$. Suppose $T$ is a multilinear operator and that

$$
\left\|T\left(f_{1}, \ldots, f_{K}\right)\right\|_{Q} \leq M_{j}\left\|f_{j}\right\|_{p} \prod_{\ell \neq j}\left\|f_{\ell}\right\|_{\infty} \quad(j=1, \ldots, K) .
$$

Then

$$
\left\|T\left(f_{1}, \ldots, f_{K}\right)\right\|_{Q} \leq \prod_{j=1}^{K}\left(M_{j}^{p / p_{j}}\left\|f_{j}\right\|_{p_{j}}\right) .
$$

Proof. We use a straightforward induction argument. If $K=2$, the corollary follows from Corollary 4 . Now assume that the corollary is true with $K$ replaced by $K-1$ for some $K \geq 3$. Interpolating the estimates $\left(\mathrm{E}_{K}\right)$ and $\left(\mathrm{E}_{j}\right)$, using Corollary 4 , gives for $1 \leq j \leq K-1$

$$
\left\|T\left(f_{1}, \ldots, f_{K}\right)\right\|_{Q} \leq M_{K}^{p / p_{K}} M_{j}^{p / q}\left\|f_{K}\right\|_{p_{K}}\left\|f_{j}\right\|_{q} \prod_{\ell \neq K, j}\left\|f_{\ell}\right\|_{\infty},
$$

where $\frac{1}{q}=\sum_{\ell=1}^{K-1} \frac{1}{p_{\ell}}$. We may assume $q<\infty$, since otherwise there is nothing to prove. Now fix a function $f_{K}$ and apply the induction hypothesis to the estimates $\left(\mathrm{F}_{j}\right), 1 \leq j \leq K-1$ (with the constants $\widetilde{M}_{j}=M_{K}^{p / p_{K}} M_{j}^{p / q}\left\|f_{K}\right\|_{p_{K}}$ ). We get

$$
\begin{aligned}
& \left\|T\left(f_{1}, \ldots, f_{K}\right)\right\|_{Q} \leq \prod_{j=1}^{K-1}\left(\widetilde{M}_{j}^{q / p_{j}}\left\|f_{j}\right\|_{p_{j}}\right) \\
& \quad=M_{K}^{p / p_{K}}\left\|f_{K}\right\|_{p_{K}} \prod_{j=1}^{K-1}\left(M_{j}^{p / p_{j}}\left\|f_{j}\right\|_{p_{j}}\right)=\prod_{j=1}^{K}\left(M_{j}^{p / p_{j}}\left\|f_{j}\right\|_{p_{j}}\right)
\end{aligned}
$$

\section{Proof of Theorem 1}

Fix a domain $\Omega$ in $\mathbf{R}^{n}, 0<|\Omega|<\infty$, and $p \in(1, \infty)$. Define a Young's function $Q$ by $Q(t)=\frac{1}{c_{1}|\Omega|}\left(\exp \left(\frac{n}{w_{n-1}} t^{p^{\prime}}\right)-1\right), t \geq 0$, where $c_{1}=c_{0}-1 \quad(>0)$ 
and $c_{0}=c_{0}(p)$ is the constant appearing in (1). Then (1) may be rewritten as

$$
\int_{\Omega} Q\left(\frac{\left|I_{\alpha}(f)(x)\right|}{\|f\|_{p}}\right) d x \leq 1
$$

which is equivalent to the norm estimate

$$
\left\|I_{\alpha}(f)\right\|_{Q} \leq\|f\|_{p} \quad\left(\forall f \in L^{p}(\Omega)\right),
$$

in view of the definition of the (Luxemburg) norm. In exactly the same way, the estimate ( 3 ) is equivalent to

$$
\left\|I_{\alpha}\left(f_{1}, \ldots, f_{K}\right)\right\|_{Q} \leq L^{-1} \cdot \prod_{j=1}^{K}\left\|f_{j}\right\|_{p_{j}} \quad\left(\forall f_{j} \in L^{p_{j}}(\Omega), 1 \leq j \leq K\right) .
$$

We will deduce (8) from (7) and Corollary 5. We have for $1 \leq j \leq K$

$$
\begin{aligned}
\left|I_{\alpha}\left(f_{1}, \ldots, f_{K}\right)(x)\right| & \leq \int_{\mathbf{R}^{n}}\left|f_{j}\left(x-\theta_{j} y\right)\right||y|^{\alpha-n} d y \prod_{\ell \neq j}\left\|f_{\ell}\right\|_{\infty} \\
& =\left|\theta_{j}\right|^{-\alpha} I_{\alpha}\left(\left|f_{j}\right|\right)(x) \prod_{\ell \neq j}\left\|f_{\ell}\right\|_{\infty} .
\end{aligned}
$$

Hence

$$
\begin{aligned}
\left\|I_{\alpha}\left(f_{1}, \ldots, f_{K}\right)\right\|_{Q} & \leq\left|\theta_{j}\right|^{-\alpha}\left\|I_{\alpha}\left(\left|f_{j}\right|\right)\right\|_{Q} \prod_{\ell \neq j}\left\|f_{\ell}\right\|_{\infty} \\
& \leq\left|\theta_{j}\right|^{-\alpha}\left\|f_{j}\right\|_{p} \prod_{\ell \neq j}\left\|f_{\ell}\right\|_{\infty} \quad(\text { by } \quad(7)) \quad(1 \leq j \leq K) .
\end{aligned}
$$

Therefore, an application of Corollary 5 gives

$$
\begin{aligned}
& \left\|I_{\alpha}\left(f_{1}, \ldots, f_{K}\right)\right\|_{Q} \leq \prod_{j=1}^{K}\left(\left|\theta_{j}\right|^{-\alpha p / p_{j}}\left\|f_{j}\right\|_{p_{j}}\right) \\
& =\prod\left(\left|\theta_{j}\right|^{-n / p_{j}}\left\|f_{j}\right\|_{p_{j}}\right) \quad(\text { since } \alpha=n / p) \\
& =L^{-1} \prod_{j=1}^{K}\left\|f_{j}\right\|_{p_{j}}
\end{aligned}
$$

with $L=\prod_{j=1}^{K}\left|\theta_{j}\right|^{n / p_{j}}$. This finishes the proof of $(8)$ and Theorem 1 .

\section{REFERENCES}

[A] D. Adams, A sharp inequality of J. Moser for higher order derivatives, Ann. of Math. (2) 128 (1988), 385-398.

[G] L. Grafakos, On multilinear fractional integrals, Studia Math. (to appear).

[KR] M. A. Krasnosel'skii and Ya. B. Rutickii, Convex functions and Orlicz spaces, Groningen, 1961.

[M] J. Musielak, Orlicz spaces and modular spaces, Springer-Verlag, New York, 1983.

[R] M. M. Rao, Interpolation, ergodicity, and martingales, J. Math. Mech. 16 (1966), 543-567.

[S] E. M. Stein, Singular integrals and differentiability properties of functions, Princeton Univ. Press, Princeton, NJ, 1970. 
[SW] E. M. Stein and G. Weiss, An introduction to Fourier analysis on Euclidean spaces, Princeton Univ. Press, Princeton, NJ, 1971.

[Y] K. Yosida, Functional analysis, 6th ed., Springer-Verlag, New York, 1980.

Department of Mathematics, Florida State University, Tallahassee, Florida 32306

E-mail address: bak@math.fsu.edu 